\title{
From the 3D Constructions of Gell-Mann's 1960 to the Higgs Field Constructions with Data of all Elementary Particles
}

\author{
Hermann Josef Scheuber
}

\section{ABSTRACT}

\begin{abstract}
In 1960 Gell-Mann completed the "Particle Zoo" with pseudo 3D constructions: a Spin-Strangeness plane and an oblique incident charge. In this way he investigated with the crossing Kaon connections (1/2 Spin, -1, 0, 1 Strangeness, -1, 0, 1 Charge) 3 quark-points with simple proper fractions. With the new media the construction can be better detected with a perpendicular charge axis as could be done with the GeoGebra 5 program. But 1960 the Quantum Mechanics didn't want the Strangeness and prevented a construction for everyman. Only experts were mathematically according to Lagrange allowed to get an idea about the real matter. But according to the Euclid Geometry 3 points lay on a circle line; if twice, then with 6 exact Quark points all other known requirements of the particle physics can be done by construction.
\end{abstract}

Keywords: Electron, Elementary particles, Gluons, Higgs field, neutrinos, Quarks.
Published Online: July 27, 2021

ISSN: $2684-4451$

DOI :10.24018/ejphysics.2021.3.4.92

Hermann Josef Scheuber*

Chemistry Department, ETHZ, Zurich, Switzerland.

(e-mail: hermann.scheuber@alumni.ethz.ch

*Corresponding Author

\section{ARGUMENTATION FOR A GEOMETRICAL CONSTRUCTION OF THE STANDARD MODEL WITH ALL ELEMENTARY PARTICLES}

It is known that the "Standard Model" (without Graviton) has 61 Particles, but all are independent as Gell-Mann did not continue his "Eightfold Way"-constructions with 3 quarks because of the "better Lagrange mathematics". But now there are 6 known quarks with the Higgs particle and new media such as the GeoGebra program and there is still no System for the Standard model. But as 1960 in the literature of "Chemie für Labor und Betrieb" (before it was overtaken by the Quantum Mechanics) I became very interested in the new particles, so I could then construct a three-dimensional model, which I studied alone after my retirement with the GeoGebra program. The Pauli principle of $-1 / 2$ and $+1 / 2$ Spin inversion (entangled quantum) could be enclosed with a circle line with a center point. The relationship (discovered by Gell-Mann) of the Quarks from Kaons with Strangeness 1 and +1 resulted in 3 definite cross points at Halfspin charge: Up- and Down Quarks, Neutrinos, Electrons) lie on circle lines (as required by Pauli). That means that the center point for the Quark circles must be found, which could be done by moving the Spin by - and $+1 / 12(=0.0833333)$. Connecting the 3 opposing 2 Pauli points through the center point gives first the 3 entangled Counter quarks and, in the center, again 3 (Quantum Chromo Dynamic)-Quarks, meaning each of the 2 Quark planes has 9 quarks. Now according to Dirac everything must be synchronized through the Higgs center point, resulting with 18 quarks and 18 Antiquarks as shown by 4 plane circle lines.

Gell-Mann's construction was also applied for the Neutrino plane: Crossing the $1 / 2$ Spin Neutrino circle by the 2 ( $\mathrm{R} \& \mathrm{~L}$ ) Zero-Kaons gives the lefthanded Electron- and the righthanded Anti-Neutrino with high precision. The left - My with $-1 / 2$ Spin and the right + Tau with $+1 / 2$ Spin are valid for the Neutrino-, Electron- \& Positron plane. Each circle line has therefore 4 controlled particles with the circle center (the My - Tau twins with the particle \& entangled counter particle).

The Positron lies on half the way from the Baryon +2 with Spin $3 / 2$ to the neutral Electro center with Spin 0 but -1 Strangeness; therefore, the Data for the Positron are $(3 / 4$, $1 / 2,+1)$ and for the Electron $(-3 / 4,1 / 2,-1 \mathrm{e})$, always (Spin, Strangeness, Charge).

6 Gluons with color \& counter color of the Quarks have to lie at the ends of the 3 Quark diagonals and 2 Gluons with even more colors lie on the Spin axis; on the + side for the upper and on the - side for the lower quarks, but all 8 lie on the Bosonic zero charged circle Spin 1, outside of the Neutrino $1 / 2$ Spin circle. The Projections of the 6 Quarks to the Bosonic circle plane are the Gravity points of the 6 triangles, so that there is no place for a fourth generation of matter. Also, the Neutron (with down quarks) has Gluon 7 but the Proton (with upper quarks) has Gluon8.

Future: As the Vector Spin of the symmetric Graviton is known as 2, then should for a three-dimensional, symmetric construction also the Vector be 2 for the Strangeness and the Charge, so that the resulting sphere tensor embraces each of the now 64 Elementary Particles.

Nonmathematical people can see therefore the Standard 
Model of the real matter direct as picture with all 61 or 64 Data [1].

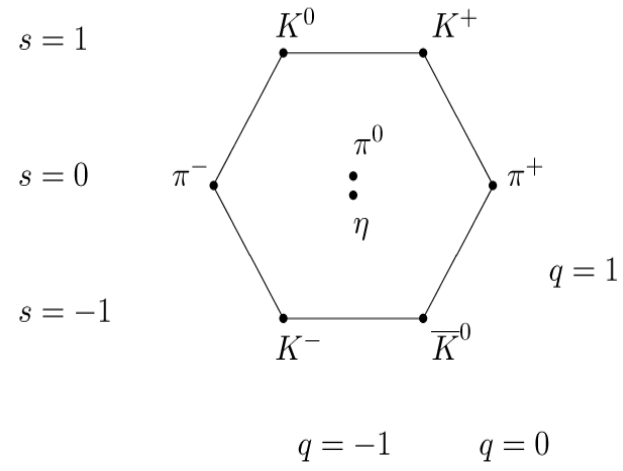

Gell-Mann's pseudo space constructions need Spin \& Strangeness ( $\mathrm{s}=1,0$, -

1) with oblique shown charge q as (Spin, Strangeness, Charge). Simple proper fraction points he got by crossing Kaon lines (Spin $1 / 2$ with Strangeness -1, 0, 1 and Charge -1, 0,1), "Quarks", prob. Gravity points.

Fig. 1. Pseudo 3D Construction of Gell-Mann for starting [2].

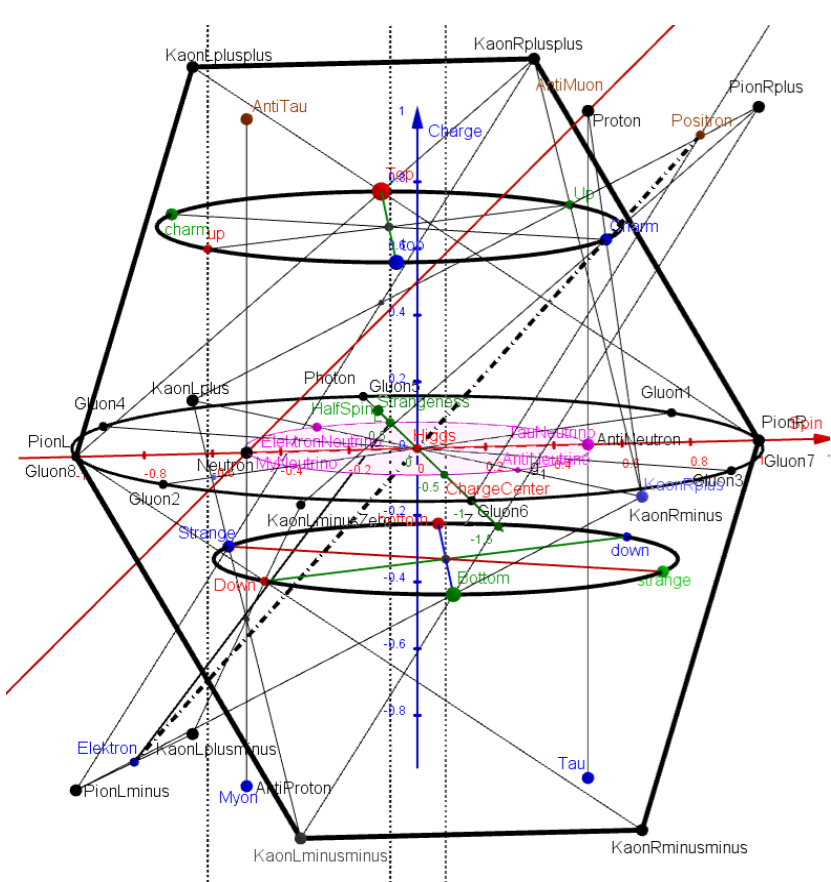

Fig. 2. Enhanced 3D horizontal construction with all 18 Quarks, (the 6 Quarks, 6 Pauli counter quarks and the 6 QCD center Quarks) with the 8 Gluons on 4 Diagonals, Positron with Electron.

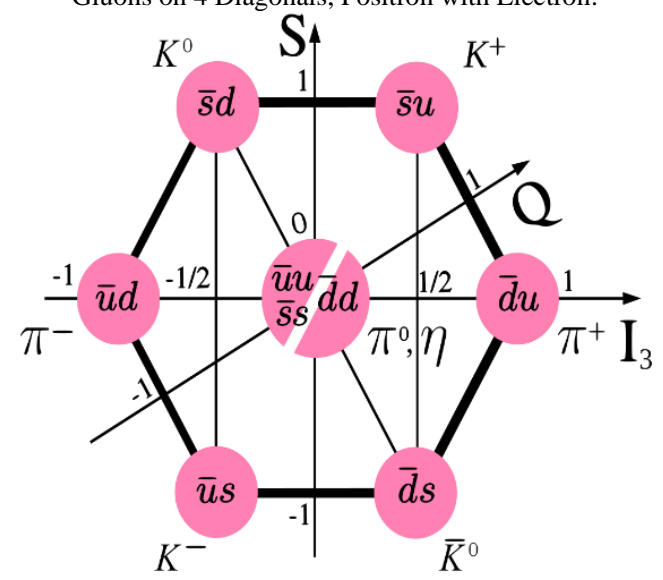

Fig. 3. Bosonic Spin1; Strangeness (1, 0, -1).

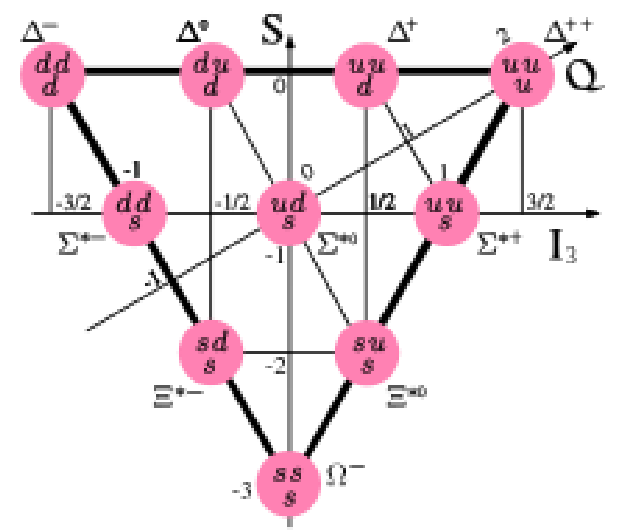

Fig. 4. Strangeness 0 until -3 Spin $-3 / 2$ bis $+3 / 2$, Charge $Q+2$ bis $-1=$ pseudo stereo on Strangeness -1 on Gell-Mann's Decuplet construction, 1 = Positron; 0 = Higgs boson; -1 = Electron (charge e, oblique shown)

\section{CONSTRUCTION PRINCIPLES}

As the directions of $\mathrm{W}+\& \mathrm{~W}$ - of the Standard Model (SM) are not known, the charge $\mathrm{W}(=\mathrm{W}+\& \mathrm{~W}-)$ can be applied. As space coordination middle point Zero: the Higgs boson is involved. The neutral Spin-strangeness plane has charge $\mathrm{W}=0$. Also, Gell-Mann used these constructions in «Eightfold Way» but with oblique incoming charge +2 from right side. From this Baryon charge $+2($ Spin $3 / 2)$ to the electric center point $(0$, strangeness $-1,0)$ lies the positron in the middle $(3 / 4,-1 / 2,+1)$.

As 3. Coordination-parameter I put the charge W perpendicularly down -up. According to the CERN-brick box always 3 Leptons have the same charge. But 3 points in a plane form a circle line. 2 points have Spin $1 / 2$ and $-1 / 2$; The strangeness is $1 / 3$ and $-1 / 3$. 1 point with Spin 0 has strangeness $2 / 3$ or $-2 / 3$. Then $U p=(1 / 2,1 / 3,2 / 3)$, Down $=(-$ $1 / 2,-1 / 3,-1 / 3)$, Charm $=(1 / 2,-1 / 3,2 / 3)$, Strange $=(-1 / 2,1 / 3$, $-1 / 3)$, Top $=(0,+2 / 3,+2 / 3)$, Bottom $=(0,-2 / 3,-1 / 3)$. According to Pauli the Spin-inverted particles lie constructively on the other side of the diameter through the center point.

Then only 6 quarks would lie on one plane; but the 3 missing particles lie in the (white) center where the 3 genetic Diagonals (of the 3 Color can be given Quantum Chromo Dynamic (QCD) as third quark in the baryons. So, there are in each of the two planes 9 Quarks definitely.

The 6 complementary "colored" Gluons on both sides lie (charge $=0$ ) on the 1 BosonSpin circle at the ends of the 3 diagonals; the 2 multiple "colored" but no charged Gluons lie at the $+\&$ - free ends of the Spin1 for the upper and lower quarks.

This is exactly the geometrical reason that the real matter does not have more than 3 generation steps.

Stereo pictures can only be seen in videos or with a corresponding stereo program (GeoGebra); otherwise only momentarily pictures are possible. According to Dirac also again but center symmetrical particles are necessary.

Therefore, there exist also at $\mathrm{W}=1 / 3 \& \mathrm{~W}=-2 / 3$ two full planes more with complementary "colors" magenta, cyanblue \& yellow.

The (left turning) Electron has according to Pauli the (right turning) Counter electron as well the (right turning) Positron (or Anti Electron) with the (left turning) Counter positron. 
The Electron plane has 4 particles: Electron, Counter electron, the pair of twins Muon (Anti Proton) \& Tau electron. The Positron plane has 4 particles: Positron, Counter positron, the pair of twins Anti Muon (Proton) \& Anti Tau. The Neutrino plane has 4 particles: Electron Neutrino, Anti Neutrino, the pair of twins Mu Neutrino, Tau Neutrino. The electromagnetic Boson, the Photon, as well the week force particle lies together with the 8 Gluons on the 1 Spin Bosonic circle. The + charged $\mathrm{W}+1$ and the - charged $\mathrm{W}-1$ Particles lie perpendicular to the vertical 1 Spin Bosonic circle.

Finally, there are 61 experimentally proven particles shown in the Higgs field Stereo picture, but no picture exists officially

If Gell-Mann could show that the 3 Quarks can be found by construction, then can also be shown that the Higgs field can be found by construction - at least for the nonmathematicians, meaning the common mob and not only the experts. Important, that the Higgs field doesn't needs nor time nor input and therefore also no Heisenberg's uncertainty - contrary to the 1960 accepted Quantum Mechanic for further experiments. But in the meantime, there are no new results be found and the new media are built for 3D space pictures, so that each particle physics student can do that.

The next construction shows exactly from step 29 to step 46 which Kaonic connections have to be taken to get the 6 crossing points Quarks on $+2 / 3$ and $-1 / 3$ planes. The 3 upper \& 3 lower Quarks lie on circle lines, but with aberrations of the centers on the Spin line of $+\&-1 / 12=0.08333$.

Therefore, the entangled counterpoints have a $1 / 6$ higher or lower Spin, e.g., instead of $1 / 2$ there are + or $-2 / 3$ or instead of 0 there are + or $-1 / 6$. The construction can be seen step by step on [4], [5]

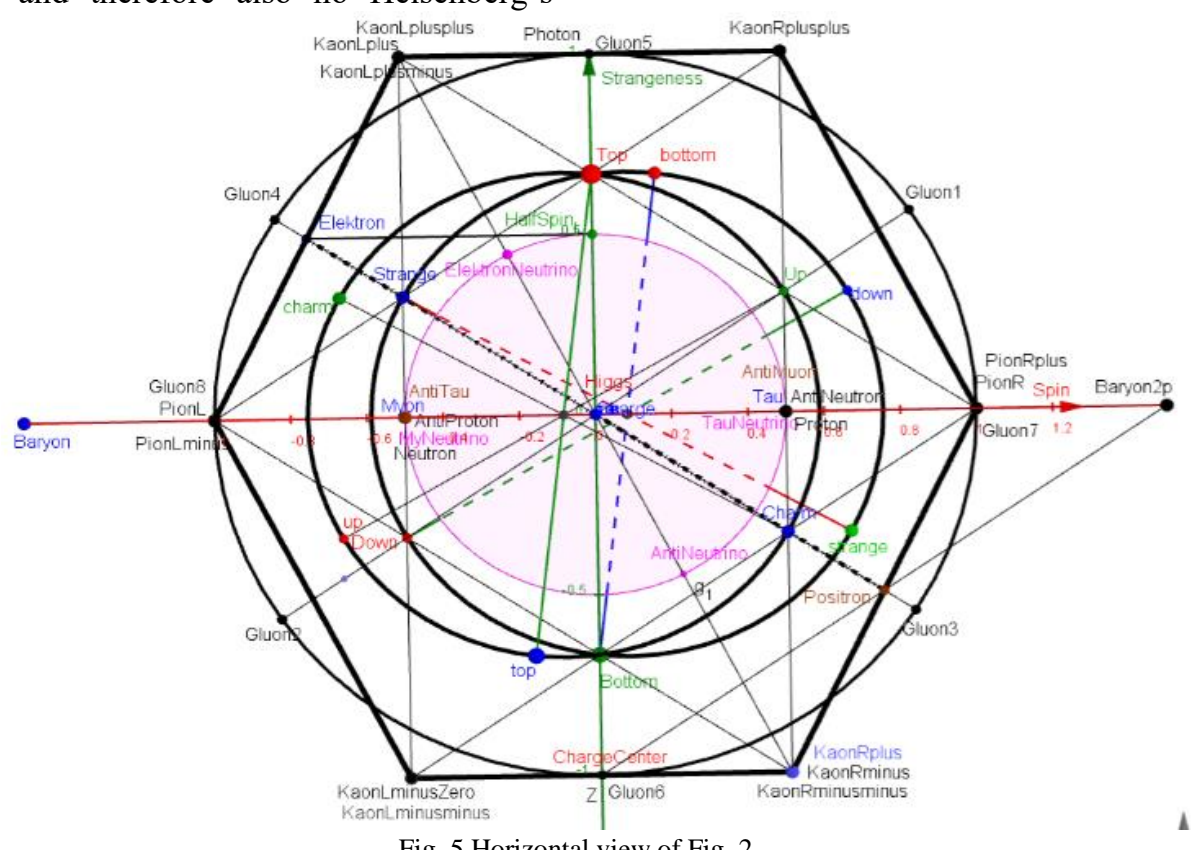

\section{CONSTRUCTIONS}

Fig. 5 Horizontal view of Fig. 2.

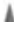

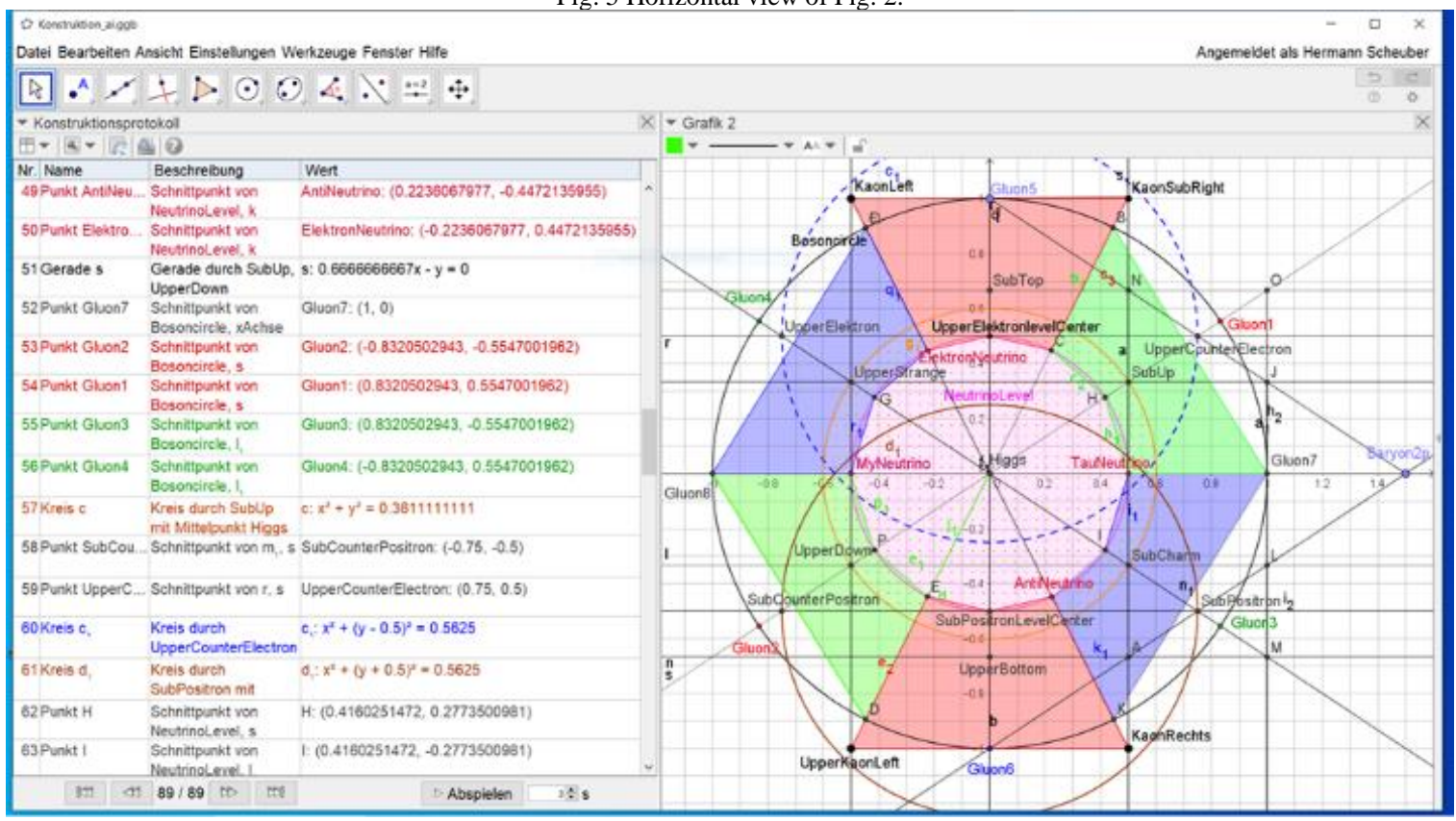

Fig. 6. The 6 quarks as projected Gravity points on the Bosonic Spin 1 plane. 
European Journal of Applied Physics www.ej-physics.org
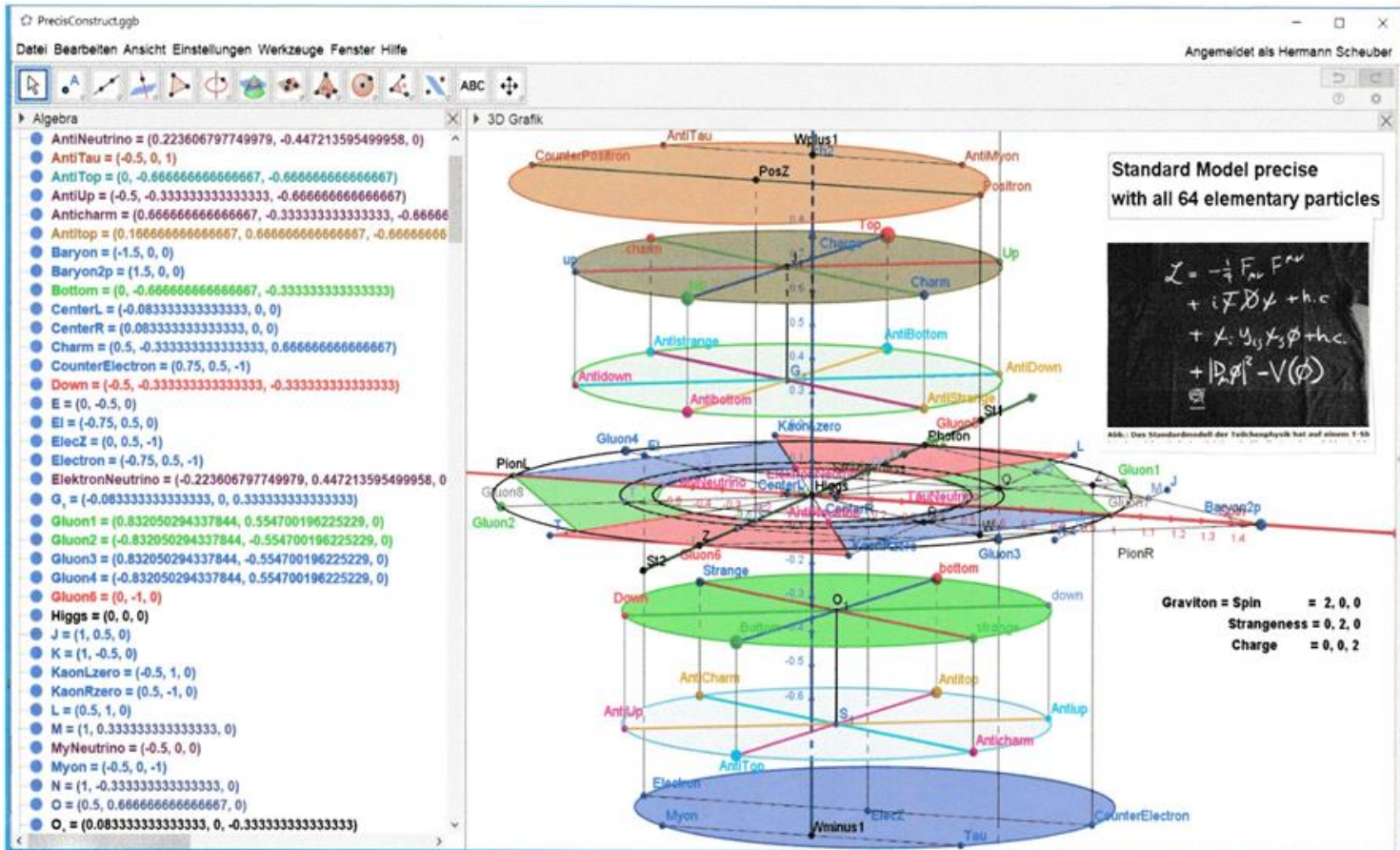

Fig. 7 After extension with Dirac there are 7 plains.

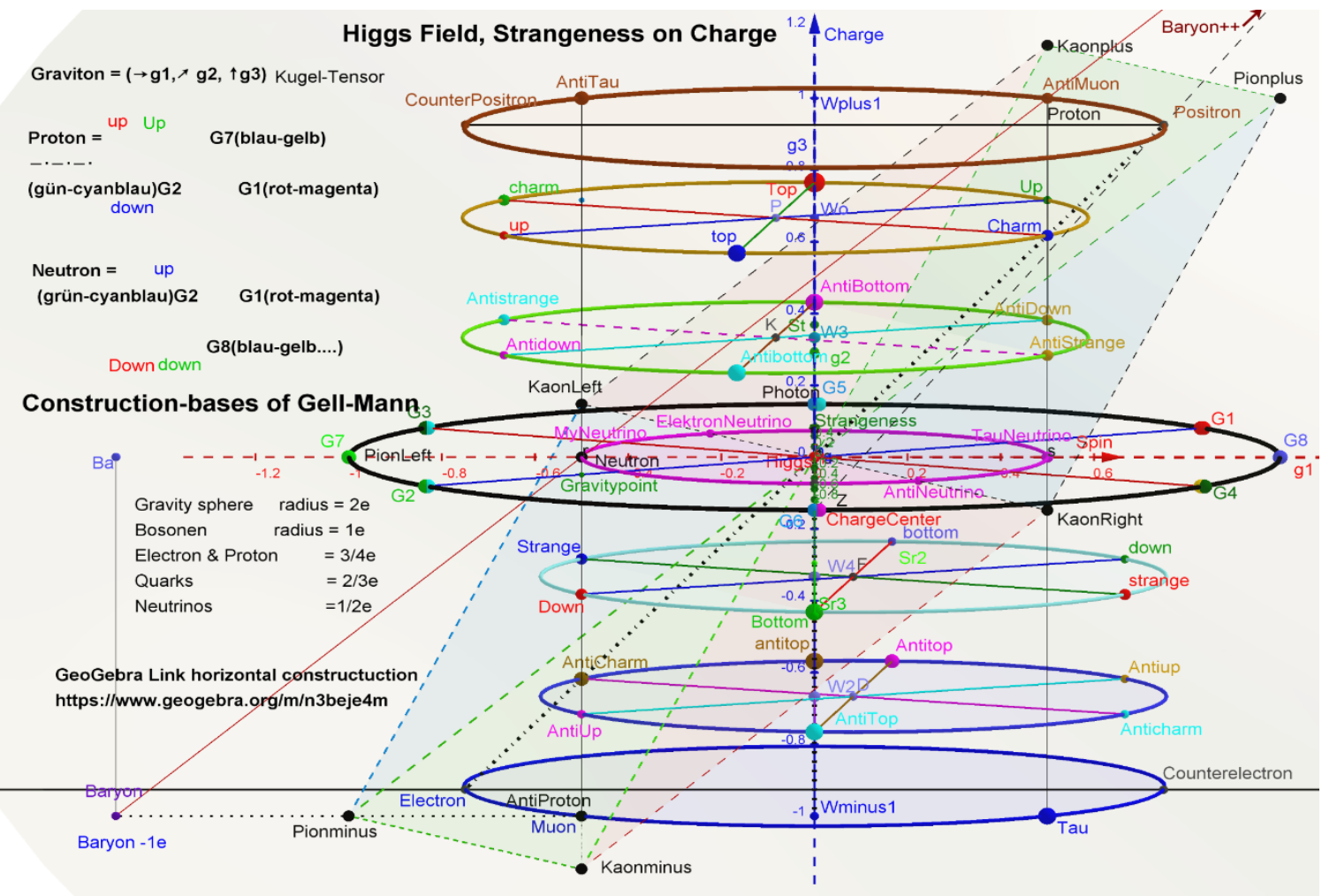

Fig. 8. The plain diameters are different with proper fractions dependent from the particles (bosons, electrons, quarks, neutrinos). 


\section{Resulting Data (Direct From the CONSTRUCTION PROTOCOL)}

\section{Data of 61 Elementary particles of the Standard Model as (Spin, Strangeness,Charge) = Higgs Field}

\begin{tabular}{|c|c|c|c|}
\hline 1) Basic-Data: & Higgs- center pa & ticle & $=(0,0,0)$ Bosons \\
\hline & Photon & & $(1,1,0)$ links drehend \\
\hline & $z^{\circ}$ & & $(1,-1,0)$ links drehend \\
\hline & $w_{+}$ & & $(0,0,+1)$ charge \\
\hline & w- & & $(0,0,-1)$ charge \\
\hline & Gluons & $\begin{array}{r}\text { red } \\
\text { blue } \\
\text { green }\end{array}$ & $\begin{array}{r}(-1,0,0) \text { antired } \\
\text { antiblue } \\
\text { antigreen }\end{array}$ \\
\hline & G6 & $\begin{array}{c}\text { red } \\
\text { antiblue }\end{array}$ & $(0,-1,0)$ \\
\hline & G4 & $\begin{array}{l}\text { green } \\
\text { antired }\end{array}$ & $(-0.83205 . ., 0.5547 . ., 0)$ \\
\hline & G2 & $\begin{array}{c}\text { blue } \\
\text { antired }\end{array}$ & $(-0.83205 . .,-0.5547 . ., 0$ \\
\hline
\end{tabular}

$(1 / 2,1 / 3,2 / 3)$

$(1 / 2,-1 / 3,2 / 3)$

Charm

Top

Down

Strange

Bottom

My-Neutrino

$(0,2 / 3,2 / 3)$

$(-1 / 2,-1 / 3,-1 / 3)$

$(-1 / 2,1 / 3,-1 / 3)$

$(0,-2 / 3,-1 / 3)$

$(-1 / 2, \quad 0,0)$

Electron-Neutrino

$(-0.2236 . ., 0.4472 \ldots, 0)$

Positron $\quad(3 / 4,-1 / 2,+1)$

Electron

$(-3 / 4,1 / 2,-1)$

Muon

Antimuon

$(-1 / 2,0,-1)$

$(1 / 2,0,+1)$

3) Anti-Quarks:

Anti-Up

$(-1 / 2,-1 / 3,-2 / 3)$

Anti-Charm

$(-1 / 2,1 / 3,-2 / 3)$

Anti-Top

Anti-Down

$(0,-2 / 3,-2 / 3)$

$(1 / 2,1 / 3,1 / 3)$

Anti-Strange

$(1 / 2,-1 / 3,1 / 3)$

Anti-Bottom

4) Future: Graviton

particle 62

particle 63

partikle 64
$(0,2 / 3,1 / 3)$

$(2, \quad 0,0)$

$(0,2,0)$

$(0,0,2)$
G7 green
red $(+1,0,0)$ antigreen antired

for down \& upper Quarks

G5 blue $(0,+1,0)$

3.Generation matter

G3 red (0.83205.., $-0.5547 . ., 0)$ antigreen

G1 green (0.83205.., $0.5547 . ., 0)$ antiblue

QCD

$\begin{array}{llll}\begin{array}{lll}\text { up } \\ \text { charm }\end{array} & (-2 / 3,-1 / 3,2 / 3) & \text { up3 } & (-1 / 12,0,2 / 3) \\ \text { top } & (-2 / 3,1 / 3,2 / 3) & \text { Charm3 } & (-1 / 12,0,2 / 3) \\ \text { down } & (-1 / 6,-2 / 3,2 / 3) & \text { top3 } & (-1 / 12,0,2 / 3) \\ \text { strange } & (2 / 3,1 / 3,-1 / 3) & \text { down3 } & (+1 / 12,0,-1) / 3) \\ \text { bottom } & (1 / 6,-1 / 3,-1 / 3) & \text { strange3 } & (+1 / 12,0,-1 / 3) \\ & & \text { bottom3 } & (+1 / 12,0,-1 / 3)\end{array}$

Tau-Neutrino $(+1 / 2,0,0)$

Anti-Neutrino $(0.2236 . .,-0.4472 \ldots, 0)$

Counter-Positron $(-3 / 4,-1 / 2,+1) \quad$ (officially Spin \& Strangeness exchanged)

Counter-Electron $(3 / 4,1 / 2,-1)$

Tauon $\quad(1 / 2,0,-1)$

Antitauon $\quad(-1 / 2,0,+1)$

\begin{tabular}{|c|c|c|c|}
\hline Anti-up & $(2 / 3,1 / 3,-2 / 3)$ & Antiup3 & $\begin{array}{l}(+1 / 12,0,-2 / 3) \\
\text { Antigreen }\end{array}$ \\
\hline Anti-charm & $(2 / 3,-1 / 3,-2 / 3)$ & Anticharm3 & $\begin{array}{l}(+1 / 12,0,-2 / 3 \\
\text { Antiblue }\end{array}$ \\
\hline Anti-top & $(1 / 6,2 / 3,-2 / 3)$ & Antitop3 & $\begin{array}{l}(+1 / 12,0,-2 / 3 \\
\text { Antired }\end{array}$ \\
\hline Anti-down & $(-2 / 3,-1 / 3,1 / 3)$ & Antidown3 & $\begin{array}{l}(-1 / 12,0,1 / 3) \\
\text { Antired }\end{array}$ \\
\hline Anti-strange & $(-2 / 3,1 / 3,1 / 3)$ & Antistrange3 & $\begin{array}{l}(-1 / 12,0,1 / 3) \\
\text { Antiblue }\end{array}$ \\
\hline Anti-bottom & $(-1 / 6,-2 / 3,1 / 3)$ & Antibottom 3 & $\begin{array}{l}(-1 / 12,0,1 / 3) \\
\text { Antigreen }\end{array}$ \\
\hline
\end{tabular}

no Antiparticle ! 


\section{Commons:Deletion requests/Files from User:Hsch31 round 4}

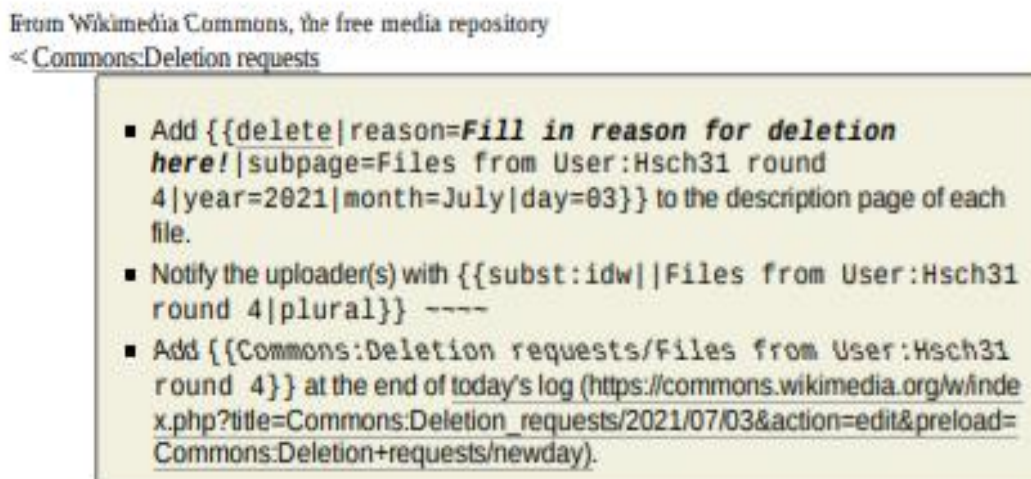

- Notify the uploader(s) with $\{$ \{subst: idw||Files from User: Hsch31 round $4 \mid$ plural $\}\}$....

- Add \{ \{Commons:Deletion requests/files from User:Hsch31 round 4 \} \} at the end of today's log (https://commons. wikimedia. org/Wfinde x.php?title=Commons:Deletion_requests/2021/07/03\&action=edit\&preload= Commons.Deletion+requesis/newday).

\section{Files from User:Hsch31 round 4}

- File:61 Elementarteilchen vertikal.png

- File:61 Elementarteilchen horizontal.png

- File:Zum Standardmodell 5.PNG

- File:Zum Standardmodell 1.png

- File:Zum Standardmodell 7hpong

- File:Standard Model horizontal of the real matter Construction with Graviton.PNG

- File:Standard Model vertical of the real matter Construction with Graviton.PNG

- File:Bosans Neutrinas level real Canstruction pog

- File:CERN-Standard Model for nonmathematic people.pdt

- File:Vergleich der verschiedenen Darstellungen des Standard Modells.pdt

- File:How to get the Standardmodel System.pdf

- File.Hsch31 im ReseauchGate.PNG

- File:3Punkte-ein Kreis.png - just mentioned for completeness, this one has its own open deletion request

Fourth round after Commons:Deletion requests/Files of User:Hsch31 and Commons:Deletion requests/More files of User:Hsch31 and Commons:Deletion requests/Even more files from User:Hsch31. Same reason again: No educational value, zero chance that any of these will ever appear in an article. Some of them are re-uploads or nearly identical with earlier deleted uploads. Some of them are attempts to write "articles" on commons. File:Hsch31 im ResearchGate.PNG has a problematic licensing status as the user specified "nut intem, nicht zur Veroffentlichung" ("only intemal, not for publication") which seems to be incomparible with CC.

Does this count as vandalism? If not, is there a different way to stop these continuous nonsense-uploads? Discussion with the user across multiple platfoums had no effect.-mflb (talk) 07:21, 13 March 2021 (UTC)

\footnotetext{
The user added File:Construction of the Higgs field with all the Data of the 61 Elementary Particles according to Gell.pdl and File:Higgs field construction of the Elementary particles.pdf and File:Link Higgsfieid Protokoll construction of the particles.pdf in the meantime. And it's again all the same nonsense. $-m$ tb (talk) 13:26, 18 May 2021 (UTC)
}

Retrieved from Titps/icommons, wakimedia.org/windex.pho?

title $=$ Commons Deletion_requests/Fies_from_User.Hsch31_ round_48ddd=561454356"

This page was last edited on 18 May 2024, at 13:26.

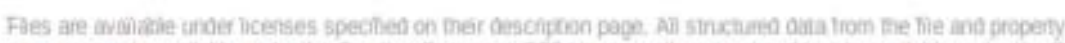

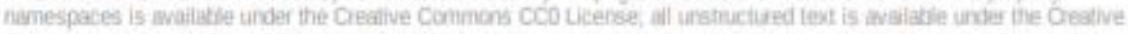
Commons Atibution-Sharedilke License, adisional terms may apply. By ising this sile, you apree to the Tems of use and the Pilvacy Palcy 


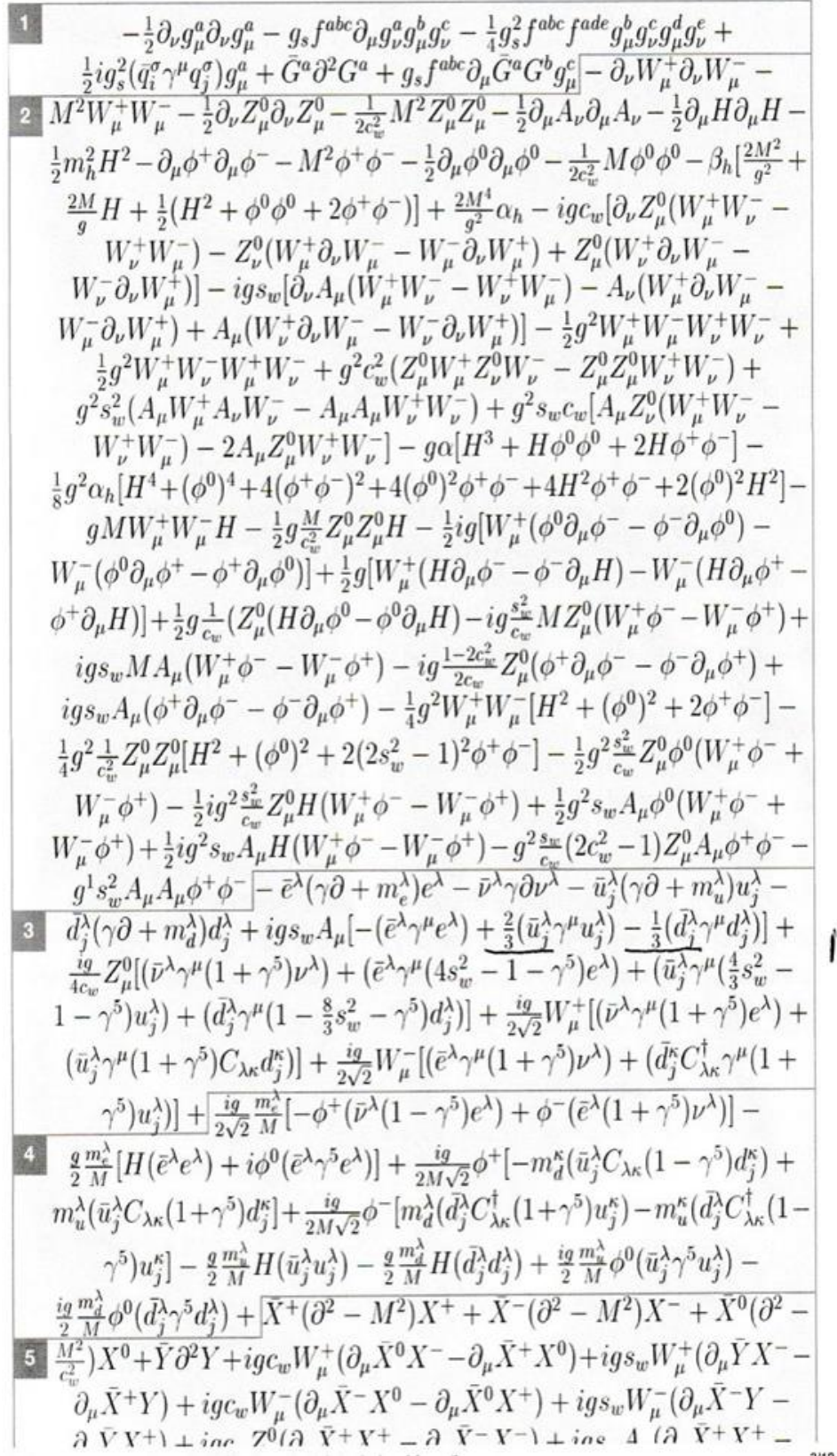

Fig.9. After consolidation with 19 constants, the mathematicians formulate with Lagrange, the nonmathematicians have nothing than the story about Higgs from Alice, Bob, and the party [3].

\section{CONCLUSION}

Until now a full construction with all Elementary particles together is missing. The Quantum Mechanic needs 19 constants for the Lagrangian formula.

To get the 6 Quarks as proper 3D fraction points (Gravity points of triangles), about the same number of "right points are necessary.

As there are more nonmathematicians, a construction for everybody is important. As long as this construction is by experiment not really wrong it is not justified to delete it as shown below. Besides it is not fair that only experts keep the knowledge without getting the Information to the mob, as done with the exaggerated "God particle"

Why was shown in the Wikipedia already a construction of a 4 generation quarks? Perhaps because a real physicist did it and not only an old man.

As there are no other information the references are limited.

\section{REFERENCES}

[1] H. Scheuber, Standard Model Picture with 61 Elementary Particles, Ijamtp Vol. 4, No. 2, 2018, pp. 42-49. doi: 10.11648/j.ijamtp.20180402.12.

[2] Eightfold Way https://de.wikipedia.org/wiki/Eightfold_Way.

[3] Mathematic formula https://www.symmetrymagazine.org/article/thedeconstructed-standard-model-equation.

[4] Step by step in youtube video Konstruktion Standardmodell, Konstruktion der Quarks und des Standardmodells.

[5] GeoGebra Link https://www.geogebra.org/m/ryqefewm also Step by step possible handling.

[6] Commons:Deletion requests/Files from User:Hsch31 round 4 Wikimedia Commons. 